\title{
Aberrant methylation of CDH11 predicts a poor outcome for patients with bladder cancer
}

\author{
YING-LI LIN $^{1 *}$, SHI-LIANG GUI ${ }^{2 *}$ and JIAN-GUO MA ${ }^{3}$ \\ ${ }^{1}$ Department of Urology, Affiliated Xuzhou Hospital of Jiangsu University, Xuzhou Cancer Hospital, Xuzhou, Jiangsu 221000; \\ ${ }^{2}$ Department of Urology, First Hospital of Jiamusi University, Jiamusi, Heilongjiang 154000; \\ ${ }^{3}$ Department of Urology, Third Hospital of Hebei Medical University, Shijiazhuang, Hebei 050051, P.R. China
}

Received July 31, 2014; Accepted April 14, 2015

DOI: $10.3892 / \mathrm{ol} .2015 .3337$

\begin{abstract}
DNA methylation is one of the major mechanisms via which tumor suppressor gene inactivation occurs. For example, hypermethylation of the promoter region of cadherin 11 (CDH11), a novel tumor suppressor gene, frequently occurs in human cancer. In the current study, the methylation status of CDH11 was investigated in bladder cancer tissue samples, and the correlation with clinicopathological features and patient outcome was assessed. The methylation status of CDH11 was detected in 146 bladder cancer tissues and 37 normal bladder epithelial tissues using methylation-specific polymerase chain reaction (PCR). In addition, CDH11 mRNA expression levels were examined by quantitative PCR. Subsequently, associations between $\mathrm{CDH} 11$ methylation and specific clinicopathological characteristics, as well as patient outcome, were analyzed. Aberrant CDH11 promoter hypermethylation was detected in $63.0 \%$ (92/146) of bladder cancer tissues, however, no CDH11 methylation was identified in the control samples; this difference was significant $(\mathrm{P}<0.05)$. Furthermore, CDH11 mRNA expression levels were significantly lower in the tumor samples with methylated CDH11 compared with the normal bladder epithelium and tumor samples with unmethylated CDH11 $(\mathrm{P}<0.05)$. When the methylation status of CDH11 was correlated with the clinicopathological features, it was identified that CDH11 methylation was significantly associated with poor differentiation $(\mathrm{P}=0.0440)$, an advanced disease stage $(\mathrm{P}=0.0350)$, a larger tumor size $(\mathrm{P}=0.0013)$ and multiple tumors $(\mathrm{P}=0.0390)$. In addition, patients with methylated CDH11 exhibited significantly poorer outcomes than patients with unmethylated CDH11 ( $\mathrm{P}=0.0004)$. Furthermore, multivariate Cox proportional hazard analysis indicated that
\end{abstract}

Correspondence to: Professor Jian-Guo Ma, Department of Urology, Third Hospital of Hebei Medical University, 139 Ziqiang Road, Shijiazhuang, Hebei 050051, P.R. China

E-mail: mjg2014sjz2@163.com

*Contributed equally

Key words: bladder cancer, methylation, cadherin 11
CDH11 methylation was independently associated with a poor outcome in the patients with bladder cancer, with a relative risk of mortality of $6.852(\mathrm{P}=0.0082 ; 95 \%$ confidence interval, 3.461-16.177). The current findings indicate that aberrant CDH11 methylation frequently occurs in bladder cancer, and correlates with malignant behavior and poor outcome. Thus, CDH11 methylation status may be used as an independent prognostic biomarker for patients with bladder cancer.

\section{Introduction}

Bladder cancer is one of the most frequently diagnosed malignancies and is a common cause of cancer-related mortality worldwide $(1,2)$. In the United States in 2013, 72,570 new cases of bladder cancer were diagnosed and 15,210 mortalities were ascribed to this malignancy (3). At diagnosis, $>70 \%$ of cases of bladder cancer are determined to be superficial transitional cell carcinomas, although the majority of these tumors relapse following transurethral resection. Furthermore, $\sim 15 \%$ will progress to muscle invasive disease $(4,5)$. However, it is estimated that the morbidity rate of bladder cancer will increase in the future due to changes in the exposure to bladder cancer risk factors, as well as the aging global population. In addition, the survival rate of affected patients is decreased with tumor progression, despite the advancement of surveillance and treatment strategies (6-10). Conventional clinicopathological parameters are commonly used to predict the course of bladder cancer, however, none are able to accurately predict the prognosis of the majority of tumors. Thus, the development of reliable prognostic biomarkers for bladder cancer remains an important challenge $(11,12)$.

It is known that bladder cancer, similar to other types of human tumor, arises from accumulated genetic and epigenetic changes that result in the inactivation of tumor suppressor genes or the activation of proto-oncogenes (13). The epigenetic silencing of tumor suppressor genes is of note in treatment and diagnosis, as epigenetic changes may be reversed to restore gene function, as well as be applied as useful biomarkers (14). For example, aberrant DNA methylation is the most common epigenetic change in human malignancies, and may be used as a diagnosis, surveillance and prognostic biomarker, particularly when the methylation silences tumor suppressor genes (15). In recent years, an association between cadherin 11 (CDH11) 
expression and human tumors has been proposed (16-19). CDH11 is a member of the cadherin superfamily, a family of calcium-dependent intercellular adhesion molecules that are crucial in cell adhesion, proliferation and invasion. Recent studies have demonstrated that CDH11 functions as a tumor suppressor gene, with the inactivation of $\mathrm{CDH} 11$ associated with the malignant behavior of various types of human tumor (16-19). The human CDH11 gene is located on chromosome 16q22.1 and is frequently silenced by promoter methylation in tumors. It is known that six classical cadherin family members, $\mathrm{CDH} 1, \mathrm{CDH} 3, \mathrm{CDH} 5, \mathrm{CDH} 8, \mathrm{CDH} 11$ and $\mathrm{CDH} 13$, are located at chromosome 16q22.1-16q24.3, as a feature termed a six-cadherin cluster, and each member has similar functions (18). In our previous studies, it was demonstrated that aberrant methylation of CDH13 is a frequent event in bladder cancer and may be applied as a useful biomarker for patients with bladder cancer (20-22). In consideration of the aforementioned findings, the present study aimed to investigate the clinical significance of CDH11 methylation in bladder cancer.

In the current study, the methylation status of CDH11 was analyzed in the tumor tissues of patients with bladder cancer by performing methylation-specific polymerase chain reaction (PCR; MSP). Subsequently, this data was correlated with common clinicopathological parameters and clinical outcomes to evaluate its clinical significance.

\section{Patients and methods}

Patients and tissue samples. The present study was performed in accordance with the Declaration of Helsinki and approved by the Ethics Committee of the Third Hospital of Hebei Medical University (Shijiazhuang, China). Written informed consent was obtained from each participant. A total of 146 tumor samples were collected during surgery at the Third Hospital of Hebei Medical University between July 2003 and July 2007, including transurethral rescetion of bladder tumor $(\mathrm{n}=95)$ and radical resection of bladder $(\mathrm{n}=51)$. The criteria for the enrollment of patients with bladder cancer were as follows: i) Histopathological diagnosis of bladder transitional cell carcinoma for the first time; ii) no other malignant tumors; iii) no anticancer therapy received prior to surgery; and iv) availability of sufficient clinicopathological and follow-up data (21). Tumor diagnosis, staging, treatment and follow-up were performed according to the European Association of Urology (EAU) Working Group on Oncological Urology: Guidelines on bladder cancer (23-25). In addition, healthy bladder epithelial tissues were obtained by biopsy from 37 inpatients with bladder calculi and used as the controls; these tissues were pathologically examined to exclude the possibility of incidental tumors. Furthermore, all control patients had no history of malignant tumors and had not previously received anticancer therapy. All tissue samples were immediately frozen in liquid nitrogen and stored at $-80^{\circ} \mathrm{C}$ until use. The clinicopathological and demographic characteristics of the patients with bladder cancer are summarized in Table I.

DNA extraction, bisulfite modification and MSP. Genomic DNA was extracted from the preserved frozen tissue samples using a DNeasy ${ }^{\circledR}$ Tissue kit (Qiagen, Inc., Valencia, CA, USA), according to the manufacturer's instructions. The extracted DNA was treated with bisulfite to convert unmethylated cytosines to uracils prior to MSP using an EpiTect ${ }^{\circledR}$ Bisulfite kit (Qiagen, Inc.), in accordance with the manufacturer's instructions and our previous study (21). The methylation status of the promoter region of CDH11 was examined by performing MSP, using primers specific for methylated and unmethylated CDH11 sequences. Each PCR reaction was carried out in a total volume of $25 \mu \mathrm{l}$, including: 100 ng DNA template and $0.2 \mu \mathrm{l}(5 \mathrm{U} / \mu \mathrm{l})$ Takara Taq DNA polymerase (Takara, Kyoto, Japan), $0.2 \mathrm{mM}$ of each primer, $2.5 \mathrm{mM}$ magnesium chloride, $0.2 \mathrm{mM}$ of each deoxynucleotide triphosphate, $2.5 \mu 1$ 10X PCR buffer (Takara). The primers for the methylated reaction were as follows: Sense, 5'-TTATTTTTGTTATTAGCGCGTTC-3' and antisense, 5'-CCATTCACAAATCAACGACG-3', with a 123-bp amplification product. The primers for the unmethylated reaction were as follows: Sense, 5'-TTTTTA TTTTTGTTATTAGTGTGTTT-3' and antisense, 5'-TCCCAT TCACAAATCAACAACA-3', with a 128-bp amplification product (Shanghai Sangon Biological Engineering Technology and Services Co., Ltd., Shanghai, China). PCR amplification of the modified DNA samples consisted of one cycle at $94^{\circ} \mathrm{C}$ for $10 \mathrm{~min}$, followed by 41 cycles at $94^{\circ} \mathrm{C}$ for $30 \mathrm{sec}, 60^{\circ} \mathrm{C}$ for $30 \mathrm{sec}$ and $72^{\circ} \mathrm{C}$ for $30 \mathrm{sec}$ for the methylated reaction, or 41 cycles at $94^{\circ} \mathrm{C}$ for $30 \mathrm{sec}, 58^{\circ} \mathrm{C}$ for $30 \mathrm{sec}$ and $72^{\circ} \mathrm{C}$ for $30 \mathrm{sec}$ for the unmethylated reaction. A final extension reaction was then performed at $72^{\circ} \mathrm{C}$ for $5 \mathrm{~min}$. Normal lymphocyte DNA, methylated in vitro with SssI methylase (New England BioLabs, Inc., Beverly, MA, USA), was used as the methylation-positive control and normal lymphocyte DNA was used as the unmethylation-positive control (26). Water samples were included with each assay as blank controls. PCR products were separated on $2 \%$ agarose gel, stained with ethidium bromide and visualized under ultraviolet illumination. Samples were scored as methylation-positive when methylated alleles were present in the methylated DNA lane and as methylation-negative when bands were present only in the unmethylated DNA lane.

Quantitative PCR analysis. Total RNA was extracted from tissue samples using TRIzol reagent (Invitrogen Life Technologies, Carlsbad, CA, USA). The mRNA expression level of CDH11 was determined by performing quantitative PCR, as previously described (27). First-strand cDNA was synthesized from 1 ug of each purified RNA sample using ExScript RT-PCR kit (Takara). Relative CDH11 mRNA expression was calculated using the comparative cycle threshold method, with GAPDH as the internal control. The primers for CDH11 were as follows: Sense, 5'-TCGCCTGCATCGTCATTC-3'; and antisense, 5'-GGCAATATCAAAGGCTTCTGTGTC-3'. The primers for GAPDH were as follows: Sense, 5'-CGCTCT CTGCTCCTCCTGTTC-3' and antisense, 5'-ATCCGTTGA CTCCGACCTTCAC-3'. The PCR conditions included a denaturation step of $95^{\circ} \mathrm{C}$ for $2 \mathrm{~min}$, followed by 40 cycles at $95^{\circ} \mathrm{C}$ for $30 \mathrm{sec}, 60^{\circ} \mathrm{C}$ for $30 \mathrm{sec}$ and $72^{\circ} \mathrm{C}$ for $2 \mathrm{~min}$, and a final elongation step of $72^{\circ} \mathrm{C}$ for $10 \mathrm{~min}$.

Statistical analysis. Fisher's exact test was used to assess the difference in CDH11 methylation status between bladder cancer samples and controls, and a $\chi^{2}$ test was performed to determine the association between CDH11 methylation status 
Table I. Association between CDH11 methylation and the clinicopathological features of bladder cancer $(n=146)$.

\begin{tabular}{|c|c|c|c|c|}
\hline Variable & Patients, $\mathrm{n}$ & Unmethylated CDH11, n (\%) & Methylated CDH11, n (\%) & P-value \\
\hline \multicolumn{5}{|l|}{ Age, years } \\
\hline$\leq 65$ & 54 & $22(40.7)$ & $32(59.3)$ & \multirow[t]{2}{*}{0.4716} \\
\hline$>65$ & 92 & $32(34.8)$ & $60(65.2)$ & \\
\hline \multicolumn{5}{|l|}{ Gender } \\
\hline Male & 102 & $37(36.3)$ & $65(63.7)$ & \multirow[t]{2}{*}{0.7862} \\
\hline Female & 44 & $17(38.6)$ & $27(61.4)$ & \\
\hline \multicolumn{5}{|l|}{ Tumors } \\
\hline Single & 52 & $25(48.1)$ & $27(51.9)$ & \multirow[t]{2}{*}{0.0390} \\
\hline Multiple & 94 & $29(30.9)$ & $65(69.1)$ & \\
\hline \multicolumn{5}{|c|}{ Tumor diameter, $\mathrm{cm}$} \\
\hline$\leq 3$ & 83 & $40(48.2)$ & $43(51.8)$ & \multirow[t]{2}{*}{0.0013} \\
\hline$>3$ & 63 & $14(22.2)$ & $49(77.8)$ & \\
\hline \multicolumn{5}{|l|}{ Tumor shape } \\
\hline Papillary & 97 & $37(38.1)$ & $60(61.9)$ & \multirow[t]{2}{*}{0.6834} \\
\hline Non-papillary & 49 & $17(34.7)$ & $32(65.3)$ & \\
\hline \multicolumn{5}{|l|}{ Grade } \\
\hline $\mathrm{G}_{1}-\mathrm{G}_{2}$ & 90 & $39(43.3)$ & $51(56.7)$ & \multirow[t]{2}{*}{0.0440} \\
\hline $\mathrm{G}_{3}$ & 56 & $15(26.8)$ & $41(73.2)$ & \\
\hline \multicolumn{5}{|l|}{ Stage } \\
\hline $\mathrm{T}_{\mathrm{a}}-\mathrm{T}_{1}$ & 95 & $41(43.2)$ & $54(56.8)$ & \multirow[t]{2}{*}{0.0350} \\
\hline $\mathrm{T}_{2}-\mathrm{T}_{4}$ & 51 & $13(25.5)$ & $38(74.5)$ & \\
\hline
\end{tabular}

CDH11, cadherin 11.

and clinicopathological features. Furthermore, the difference in CDH11 mRNA expression between the controls, patients with methylated CDH11 and patients with unmethylated CDH11 was analyzed by one-way analysis of variance. Kaplan-Meier survival analysis and log-rank tests were performed to assess the difference in overall survival between patients with methylated and unmethylated CDH11. In addition, the multivariate Cox proportional hazard model analysis was applied to determine the independent prognostic effect of CDH11 methylation. All statistical analyses were performed using SAS software (version 8.0; SAS Institute, Cary, NC, USA) and two-sided $\mathrm{P}<0.05$ was considered to indicate a statistically significant difference.

\section{Results}

In the present study, the promoter hypermethylation status of CDH11 was determined in bladder cancer tissues and normal bladder epithelial tissues using MSP. It was identified that the CDH11 promoter was hypermethylated in $63.0 \%(92 / 146)$ of bladder cancer tissue samples, however, no methylation was detected in the control samples. Thus, CDH11 promoter hypermethylation occurred significantly more frequently in the bladder cancer tissues than in the normal bladder epithelial tissues $(\mathrm{P}<0.05$; Fig. 1).

To clarify that hypermethylation of the CDH11 promoter region is correlated with the inactivation of its gene expression, quantitative PCR was performed to detect the mRNA

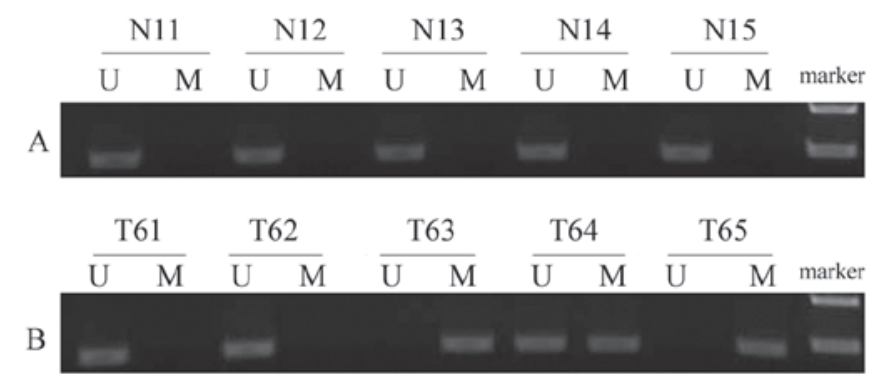

Figure 1. Representative cadherin 11 (CDH11) methylation status of (A) normal bladder epithelial tissues and (B) bladder cancer tissues, as determined by performing methylation-specific polymerase chain reaction. Samples T63, T64 and T65 exhibited hypermethylation of the CDH11 promoter. U, unmethylated; M, methylated.

expression levels of CDH11 in the bladder cancer and normal bladder epithelial samples. The tumor samples were divided into two groups, as samples with methylated or unmethylated CDH11. It was identified that CDH11 mRNA expression was similar in the tumor samples with unmethylated CDH11 and in the normal bladder epithelium. However, CDH11 mRNA expression was significantly lower in the tumor samples with methylated CDH11 compared with the normal bladder epithelium and tumor samples with unmethylated CDH11 $(\mathrm{P}<0.05$; Fig. 2). These results indicate that aberrant $\mathrm{CDH} 11$ promoter hypermethylation is the major mechanism by which CDH11 is inactivated in bladder cancer. 
Table II. Multivariate Cox proportional hazard analysis of potential prognostic factors for patients with bladder cancer ( $\mathrm{n}=146$ ).

\begin{tabular}{lcr}
\hline Variable & HR $(95 \%$ confidence interval) & P-value \\
\hline CDH11 methylation (methylated vs. unmethylated) & $6.852(3.461-16.177)$ & 0.0082 \\
Age $(>65$ vs. $\leq 65$ years) & $1.312(0.625-4.171)$ & 0.6429 \\
Gender (male vs. female) & $1.125(0.467-4.239)$ & 0.7063 \\
Number of tumors (multiple vs. single) & $2.786(0.879-9.418)$ & 0.4527 \\
Tumor diameter $(>3$ vs. $\leq 3 \mathrm{~cm})$ & $3.054(0.796-10.587)$ & 0.3786 \\
Shape (non-papillary vs. papillary) & $3.613(0.917-11.028)$ & 0.1891 \\
Grade $\left(\mathrm{G}_{3}\right.$ vs. $\left.\mathrm{G}_{1}-\mathrm{G}_{2}\right)$ & $4.317(0.936-15.068)$ & 0.0544 \\
Stage $\left(\mathrm{T}_{2}-\mathrm{T}_{4}\right.$ vs. $\left.\mathrm{T}_{\mathrm{a}}-\mathrm{T}_{1}\right)$ & $4.963(2.567-14.622)$ & 0.0357
\end{tabular}

HR, hazard ratio; CDH11, cadherin 11.

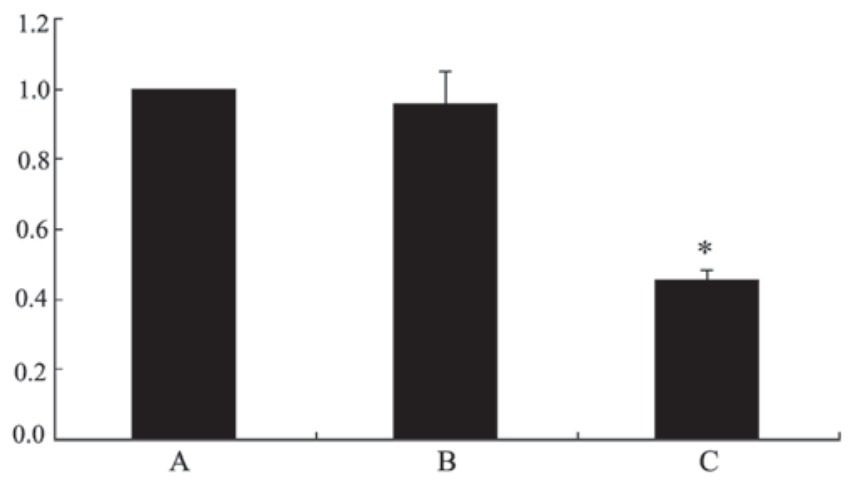

Figure 2. Cadherin 11 (CDH11) mRNA expression in normal bladder epithelial tissues (column A), bladder tumors with unmethylated CDH11 (column B) and bladder tumors with methylated CDH11 (column C). ${ }^{*} \mathrm{P}<0.05$ : C vs. A and C vs. B.

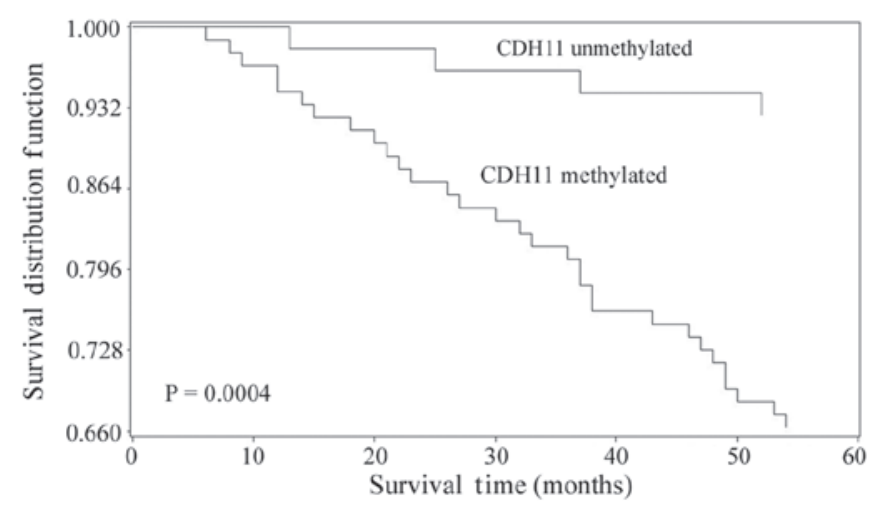

Figure 3. Kaplan-Meier survival curves for 146 samples from patients with bladder cancer according to CDH11 methylation status. Patients with methylated CDH11 exhibited significantly shorter five-year overall survival times than patients with unmethylated $\mathrm{CDH} 11(\mathrm{P}=0.0004$; log-rank test). CDH11, cadherin 11.

The aim of the present study was to evaluate the clinical significance of CDH11 methylation in bladder cancer. In addition, associations between the methylation status of CDH11 and commonly used clinicopathological parameters in bladder cancer were analyzed (Table II). It was identified that aberrant hypermethylation of CDH11 in tumor tissues was significantly associated with poor differentiation $(\mathrm{P}=0.0440)$, an advanced disease stage $(\mathrm{P}=0.0350)$, a larger tumor size $(\mathrm{P}=0.0013)$ and multiple tumors $(\mathrm{P}=0.0390)$. However, no association was detected between $\mathrm{CDH} 11$ methylation and age, gender or tumor shape. The overall survival time of the patients with bladder cancer was defined as the time from the date of diagnosis to the date of mortality from any cause or the last contact if the patient remained alive (21). Examination of the overall survival of the patients with bladder cancer according to the methylation status of $\mathrm{CDH} 11$ revealed that the patients with methylated CDH11 had poorer outcomes than the patients with unmethylated CDH11 ( $\mathrm{P}=0.0004$; Fig. 3). This finding indicated that CDH11 methylation in tumor tissue samples may be associated with a poor prognosis of bladder cancer. To further investigate the prognostic value of CDH11 methylation in bladder cancer, a multivariate Cox proportional hazard model analysis was conducted. Notably, the results indicated that CDH11 methylation may be independently associated with the poor outcome of patients with bladder cancer, exhibiting a relative risk of mortality of $6.852(\mathrm{P}=0.0082 ; 95 \%$ confidence interval, 3.461-16.177; Table II).

\section{Discussion}

The identification of prognostic and predictive markers for bladder cancer is important, as it is a heterogeneous disease with a clinical outcome that is difficult to predict (5). Morphologically and pathologically similar tumors may behave differently, therefore, it is currently not possible to accurately predict the outcome of bladder cancer following the receipt of initial adequate treatment. Clinically, it is crucial to identify patients with a high risk of mortality who require more aggressive treatment strategies and patients with a low risk of mortality requiring less intensive surveillance. Therefore, novel prognostic biomarkers must be identified and applied in addition to common clinical and pathological features. It is known that DNA methylation, one of the most common epigenetic changes, frequently occurs in various types of human cancer, including bladder cancer. Thus, the detection of aberrant DNA methylation in primary bladder tumor specimens may useful for predicting the outcome of patients with bladder cancer (14).

In the current study, the methylation status of CDH11 was investigated in bladder cancer tissues and normal bladder 
epithelium. Aberrant CDH11 promoter hypermethylation occurred in $63.0 \%(92 / 146)$ of bladder tumor samples, however, no methylation was detected in the normal bladder epithelium samples. These results indicated that aberrant methylation of CDH11 is tumor-specific, and that CDH11 may be used as potential biomarker in bladder cancer. In addition, reduced CDH11 mRNA expression was observed in the bladder tumors with aberrant promoter hypermethylation. These findings indicate that epigenetic inactivation of CDH11 by aberrant promoter hypermethylation may be crucial in the formation of bladder cancer and thus, assessment of the methylation status of CDH11 may be a useful biomarker in determining the prognosis of patients with bladder cancer. To verify this possibility, the methylation status of CDH11 was subsequently correlated with specific clinicopathological parameters of bladder cancer. Notably, aberrant methylation of CDH11 occurred frequently in the tumors with an advanced stage, high grade, larger tumor size and multiple tumors, factors that are all risk factors of a poor prognosis in bladder cancer (28). This raises the possibility that CDH11 methylation status in tumors may be applied as a prognostic biomarker that is as reliable or more reliable than currently used clinicopathological factors. To investigate this hypothesis, the overall survival of patients with bladder cancer was investigated in terms of tumor tissue CDH11 methylation status. It was identified that patients with methylated CDH11 had significantly worse outcomes than patients with unmethylated CDH11. Furthermore, multivariate Cox proportional hazard model analysis indicated that aberrant CDH11 methylation was an independent prognostic factor for the overall survival of patients with bladder cancer. Taken together, the current findings suggested that aberrant methylation of CDH11 in tumors indicates worse outcomes for patients with bladder cancer. Therefore, it is recommended that patients with bladder cancer and CDH11 methylation should receive aggressive intervention following initial curative treatment to achieve more favorable outcomes.

The findings of the present study are in accordance with previous study about CDH11 in human cancers $(16,18,19)$. DNA methylation as a potential biomarker for human cancer is of particular interest, since DNA may be collected conveniently from tissues or body fluids. In addition, DNA methylation may be reversed by demethylation agents and in the longer term may enable more individualized therapies.

The present study identified a correlation between CDH11 methylation status and the five-year overall survival time of patients with bladder cancer. However, it was limited by the small number of patients with bladder cancer that were analyzed and by the use of a single center. It is proposed that future studies continue to investigate the value of CDH11 methylation in predicting the prognosis of bladder cancer using a greater number of samples, to corroborate the findings of the present study.

In conclusion, aberrant methylation of CDH11 frequently occurs in bladder cancer and contributes to the inactivation of its expression. In addition, CDH11 methylation appears to be closely associated with malignant behavior in bladder cancer and thus, may serve as an independent prognostic biomarker. Considering the reversible nature of DNA methylation, CDH11 methylation may be a good therapeutic target for patients with bladder cancer, however, future studies are required to verify this hypothesis.

\section{Acknowledgements}

The present study was supported by the Xuzhou Medical Talented Youth Project (grant no. 2014007).

\section{References}

1. Ploeg M, Aben KK and Kiemeney LA: The present and future burden of urinary bladder cancer in the world. World J Urol 27: 289-293, 2009.

2. Parkin DM: The global burden of urinary bladder cancer. Scand J Urol Nephrol Suppl 42: 12-20, 2008.

3. Siegel R, Naishadham D and Jemal A: Cancer statistics, 2013. CA Cancer J Clin 63: 11-30, 2013.

4. Kim WJ, Kim EJ, Jeong P, et al: RUNX3 inactivation by point mutations and aberrant DNA methylation in bladder tumors. Cancer Res 65: 9347-9354, 2005.

5. Kawamoto K, Enokida H, Gotanda T, et al: p16INK4a and p14ARF methylation as a potential biomarker for human bladder cancer. Biochem Biophys Res Commun 339: 790-796, 2006.

6. Montgomery JS, Miller DC and Weizer AZ: Quality indicators in the management of bladder cancer. J Natl Compr Canc Netw 11: 492-500, 2013.

7. Shariat SF, Milowsky M and Droller MJ: Bladder cancer in the elderly. Urol Oncol 27: 653-667, 2009.

8. Mueller J, Schrader AJ, Schrader M, Schnoeller T and Jentzmik F: Management of muscle-invasive bladder cancer. Minerva Urol Nefrol 65: 235-248, 2013.

9. Pal SK, Milowsky MI and Plimack ER: Optimizing systemic therapy for bladder cancer. J Natl Compr Canc Netw 11: 793-804, 2013.

10. Kobeissi LH, Yassine IA, Jabbour ME, Moussa MA and Dhaini HR: Urinary bladder cancer risk factors: A Lebanese case-control study. Asian Pac J Cancer Prev 14: 3205-3211, 2013.

11. Ghafouri-Fard S, Nekoohesh L and Motevaseli E: Bladder cancer biomarkers: Review and update. Asian Pac J Cancer Prev 15: 2395-2403, 2014.

12. Ding MX, Wang HF, Wang JS, et al: ppGalNAc T1 as a potential novel marker for human bladder cancer. Asian Pac J Cancer Prev 13: 5653-5657, 2012.

13. Yegin Z, Gunes S and Buyukalpelli R: Hypermethylation of TWIST1 and NID2 in tumor tissues and voided urine in urinary bladder cancer patients. DNA Cell Biol 32: 386-792, 2013.

14. Kim YK and Kim WJ: Epigenetic markers as promising prognosticators for bladder cancer. Int J Urol 16: 17-22, 2009.

15. Kandimalla R, van Tilborg AA and Zwarthoff EC: DNA methylation-based biomarkers in bladder cancer. Nat Rev Urol 10: 327-335, 2013.

16. Deng Z, Niu G, Cai L, Wei $R$ and Zhao X: The prognostic significance of CD44V6, CDH11 and $\beta$-catenin expression in patients with osteosarcoma. BioMed Res Int 2013: 496193, 2013.

17. Carmona FJ, Villanueva A, Vidal A, et al: Epigenetic disruption of cadherin-11 in human cancer metastasis. J Pathol 228: 230-240, 2012.

18. Li L, Ying J, Li H, et al: The human cadherin 11 is a pro-apoptotic tumor suppressor modulating cell stemness through $\mathrm{Wnt} / \beta$-catenin signaling and silenced in common carcinomas. Oncogene 31: 3901-3912, 2012.

19. Nakajima G, Patino-Garcia A, Bruheim S, et al: CDH11 expression is associated with survival in patients with osteosarcoma. Cancer Genomics Proteomics 5: 37-42, 2008.

20. Lin YL, He ZK, Li ZG and Guan TY: Downregulation of CDH13 expression promotes invasiveness of bladder transitional cell carcinoma. Urol Int 90: 225-232, 2013.

21. Lin YL, Liu XQ, Li WP, Sun G and Zhang CT: Promoter methylation of $\mathrm{H}$-cadherin is a potential biomarker in patients with bladder transitional cell carcinoma. Int Urol Nephrol 44: 111-117, 2012.

22. Lin YL, Sun G, Liu XQ, Li WP and Ma JG: Clinical significance of $\mathrm{CDH} 13$ promoter methylation in serum samples from patients with bladder transitional cell carcinoma. J Int Med Res 39: 179-186, 2011. 
23. Oosterlinck W, Lobel B, Jakse G, et al; European Association of Urology (EAU) Working Group on Oncological Urology: Guidelines on bladder cancer. Eur Urol 41: 105-112, 2002.

24. Greene FL: The American Joint Committee on Cancer: Updating the strategies in cancer staging. Bull Am Coll Surg 87: 13-15, 2002.

25. Babjuk M, Oosterlinck W, Sylvester R, et al; European Association of Urology (EAU): EAU guidelines on non-muscle-invasive urothelial carcinoma of the bladder, the 2011 update. Eur Urol 59: 997-1008, 2011.
26. Wu Y, Zhang X, Lin L, et al: Aberrant methylation of RASSF2A in tumors and plasma of patients with epithelial ovarian cancer. Asian Pac J Cancer Prev 15: 1171-1176, 2014.

27. Nakajima G, Patino-Garcia A, Bruheim S, et al: CDH11 expression is associated with survival in patients with osteosarcoma. Cancer Genomics Proteomics 5: 37-42, 2008.

28. Kaufman DS, Shipley WU and Feldman AS: Bladder cancer. Lancet 374: 239-249, 2009. 